\title{
Exploring the Real Ground-state Structures of Molybdenum Dinitride
}

Shuyin Yu, ${ }^{*}+, \ddagger$ Bowen Huang, ${ }^{\top}$ Xiaojing Jia, ${ }^{\dagger}$ Qingfeng Zeng, ${ }^{\dagger, \ddagger}$ Artem R. Oganov, ${ }^{\ddagger, \S, /, \perp}$ Litong Zhang, ${ }^{\dagger}$ and Gilles Frapper ${ }^{*}$,

${ }^{\dagger}$ Science and Technology on Thermostructural Composite Materials Laboratory, School of Materials Science and Engineering, Northwestern Polytechnical University, Xi'an, Shaanxi 710072, China

${ }^{\ddagger}$ International Center for Materials Discovery, School of Materials Science and Engineering, Northwestern Polytechnical University, Xi'an, Shaanxi 710072, China

'IC2MP UMR 7285, Université de Poitiers - CNRS, 4, rue Michel Brunet TSA 51106 - 86073 Poitiers Cedex 9, France

${ }^{\S}$ Skolkovo Institute of Science and Technology, 3 Nobel Street, Skolkovo 143025, Russia

"Department of Geosciences, Center for Materials by Design, and Institute for Advanced Computational Science, State University of New York, Stony Brook, NY 11794-210o, USA

${ }^{\perp}$ Moscow Institute of Physics and Technology, Dolgoprudny, Moscow Region 14170o, Russia

Correspondence and requests for materials should be addressed to Gilles Frapper (email: gilles.frapper@univ-poitiers.fr)

\section{CONTENTS}

1. Crystal structure and the phonon dispersion curves of the predicted $\mathrm{C}_{2} / \mathrm{m} \mathrm{MoN}_{2}$. (Figure $\mathrm{S}_{1}$ )

2. Phonon dispersion curves of the predicted $\mathrm{P}_{3} / \mathrm{mmc}^{-}$and $\mathrm{P} 4 / \mathrm{mbm}-\mathrm{MoN}_{2}$. (Figure $\mathrm{S}_{2}$ )

3. Schematic molecular orbital diagram of $\mathrm{N}_{2}{ }^{4-}$ unit. (Figure $\mathrm{S}_{3}$ )

4. Crystal field splitting of the $\mathrm{d}_{\mathrm{Mo}}$ levels in $\mathrm{P}_{3} / \mathrm{mmc} \mathrm{MoN}_{2}$. (Figure $\mathrm{S}_{4}$ )

5. Crystal structures found by variable-composition evolutionary algorithm. (Figure $\mathrm{S}_{5}$ )

7. Comparison of the XRD patterns with experimental data. (Figure S6)

8. Calculated structural parameters, enthalpy and magnetic properties of $\mathrm{MoS}_{2}$-like $\mathrm{R}_{3} \mathrm{~m} \mathrm{MoN}_{2}$ structure (Table $\mathrm{S} 1$ )

9. Computed formation enthalpies of representative metal nitrides $M_{x} N_{y}$. (Table $S_{2}$ )

10. The calculated lattice parameters, Wyckoff positions, atomic distances, enthalpies and zero-point energies (ZPE) of the stable and metastable Mo-N structures at o GPa. (Table $\mathrm{S}_{3}$ )

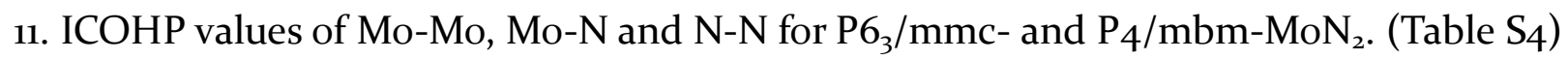


Figure $\mathrm{S}_{1}$ Crystal structure and phonon dispersion curves of the predicted $\mathrm{C}_{2} / \mathrm{m} \mathrm{MoN}_{2}$. Note that Mo is in an octahedral environment.

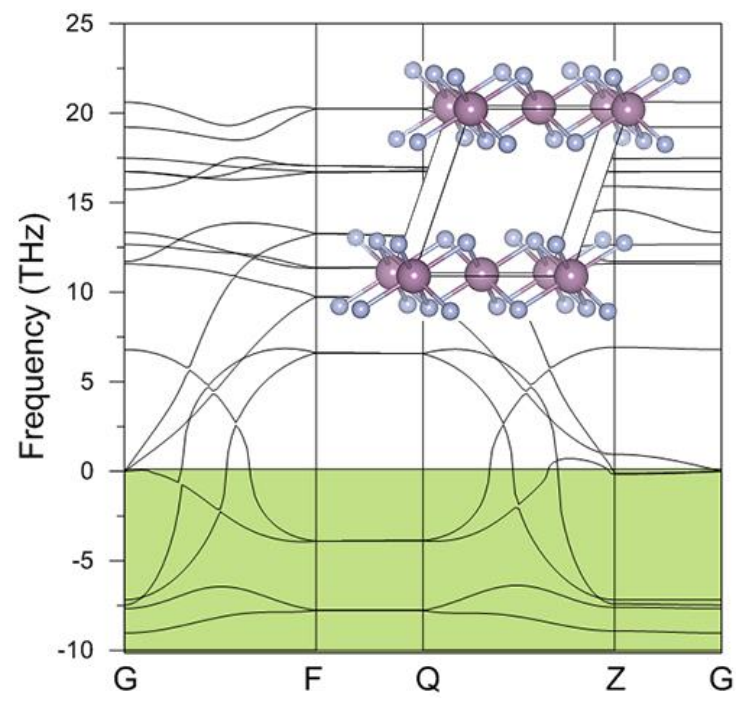

Figure $\mathrm{S}_{2}$ The calculated phonon dispersion curves of the predicted $\mathrm{MoN}_{2}$ structures. ${ }^{1} \mathrm{P} 6_{3} / \mathrm{mmc}$ at o $\mathrm{GPa}$ and ${ }^{1} \mathrm{P} 4 / \mathrm{mbm}$ at $100 \mathrm{GPa}$.
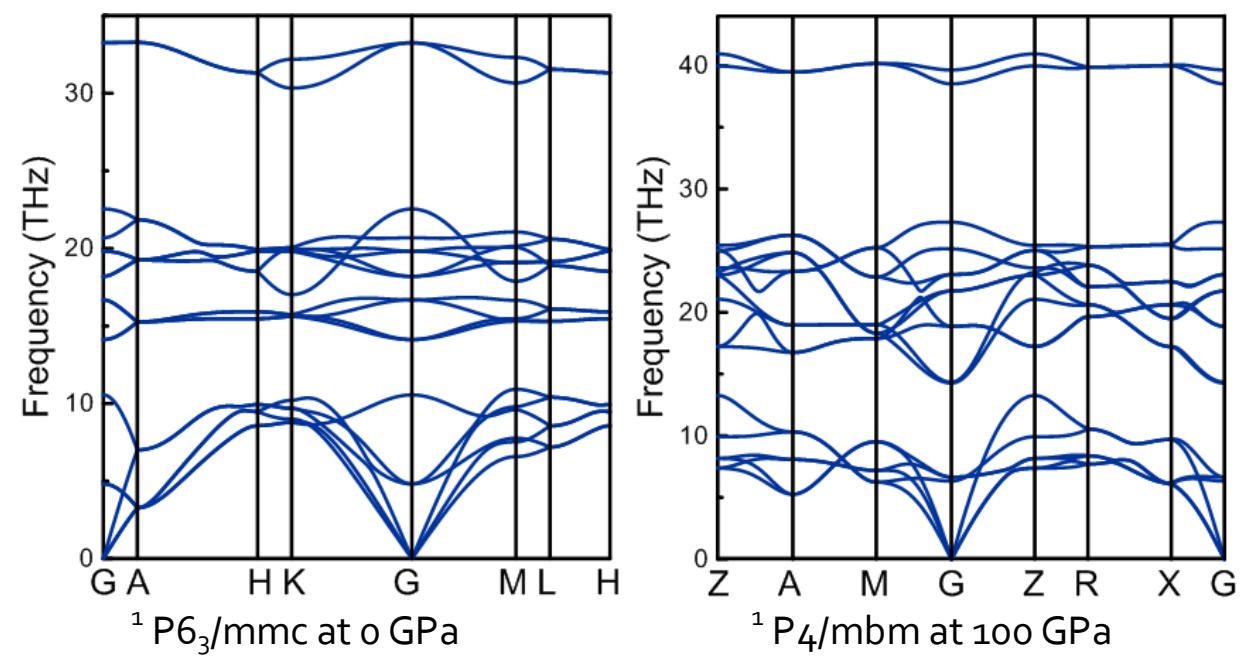
Figure $\mathrm{S}_{3}$ Schematic molecular orbital diagram of $\mathrm{N}_{2}{ }^{4-}$. The solid and dashed lines indicate the primary and secondary parentage of the orbitals, respectively $\left(2 s-2 p_{z}\right.$ mixing). Only the valence orbitals are shown. $\mathrm{N}_{2}^{4-}$ anion has 14 valence electrons and the bond order is one.

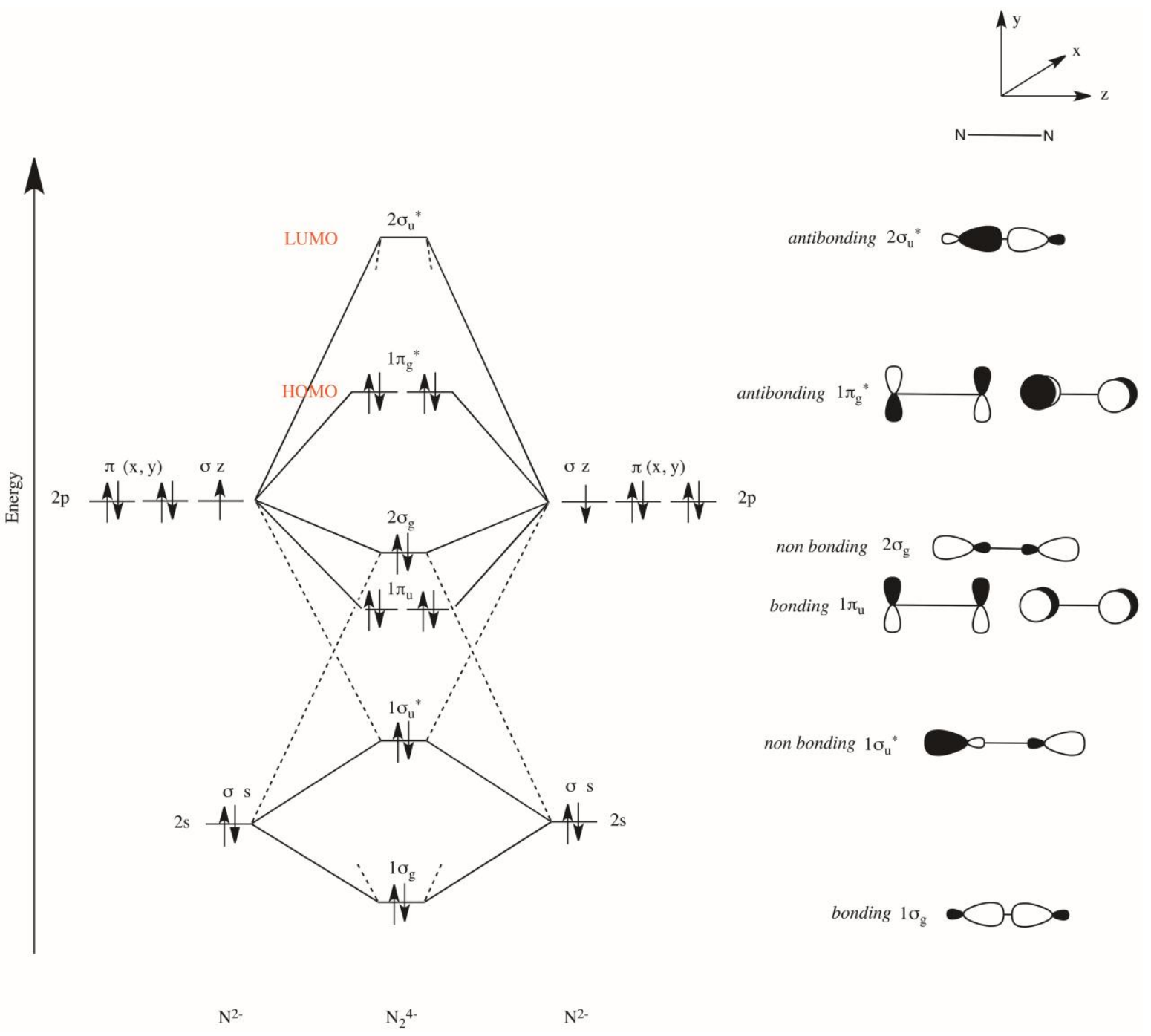


Figure $\mathrm{S}_{4}$ Crystal field splitting of the $\mathrm{d}$ levels of the Mo atoms ( $\mathrm{d}^{2}$ configuration) in a trigonal prismatic $\mathrm{MoN}_{6}$ environment of the $\mathrm{P}_{3} / \mathrm{mmc} \mathrm{MoN}_{2}$. Each Mo atom is coordinated to six $\mathrm{N}$ atoms, forming a trigonal prismatic $\mathrm{MoN}_{6}$ environment.

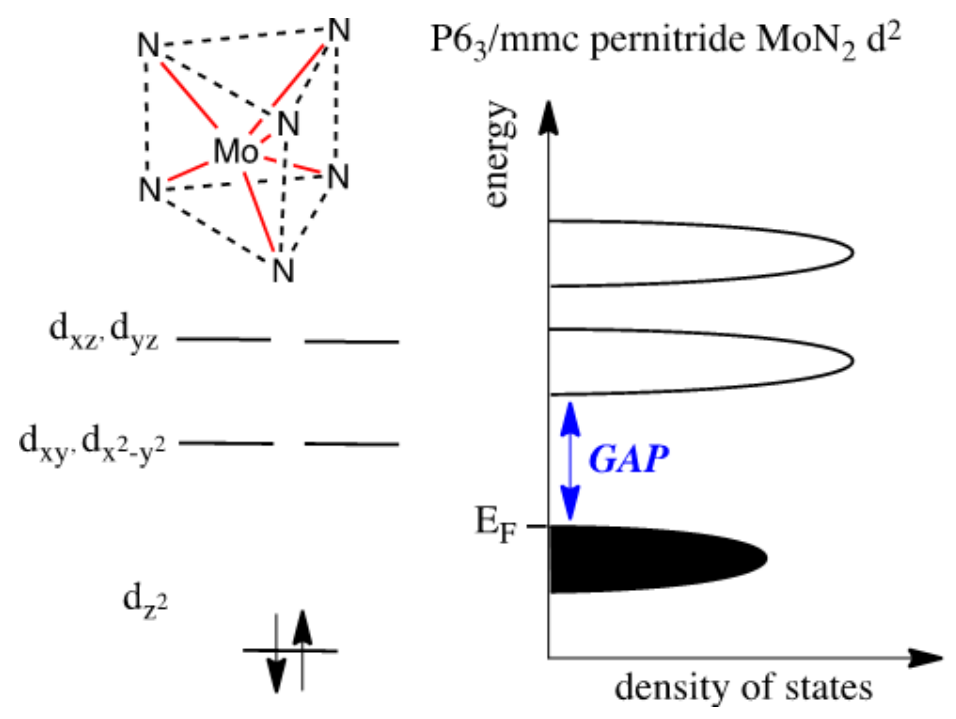

Figure $\mathrm{S}_{5}$ Crystal structures of the $\mathrm{Mo}_{x} \mathrm{~N}_{y}$. The big purple and small blue spheres represent $\mathrm{Mo}$ and $\mathrm{N}$ atoms, respectively.
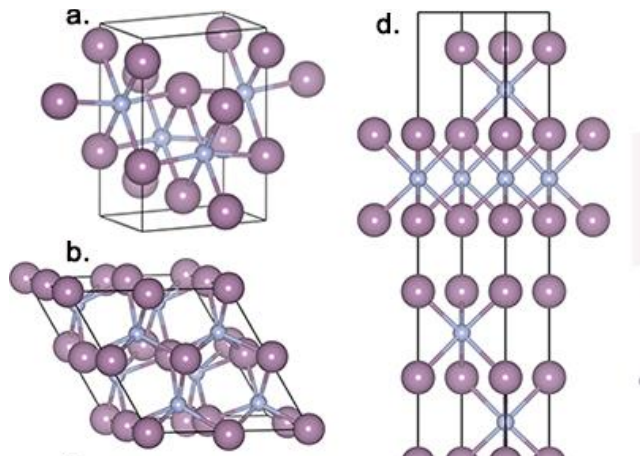

a. $\mathrm{Pmmn}-\mathrm{Mo}_{2} \mathrm{~N}$

b. $\mathrm{P6}_{3} \mathrm{mc}-\mathrm{MoN}$

c. Imm2-- $\mathrm{Mo}_{4} \mathrm{~N}_{3}$

d. $\mathrm{R}-3 \mathrm{~m}-\mathrm{Mo}_{3} \mathrm{~N}_{2}$

e. $\mathrm{P}_{3} / \mathrm{m}-\mathrm{Mo}_{5} \mathrm{~N}_{6}$
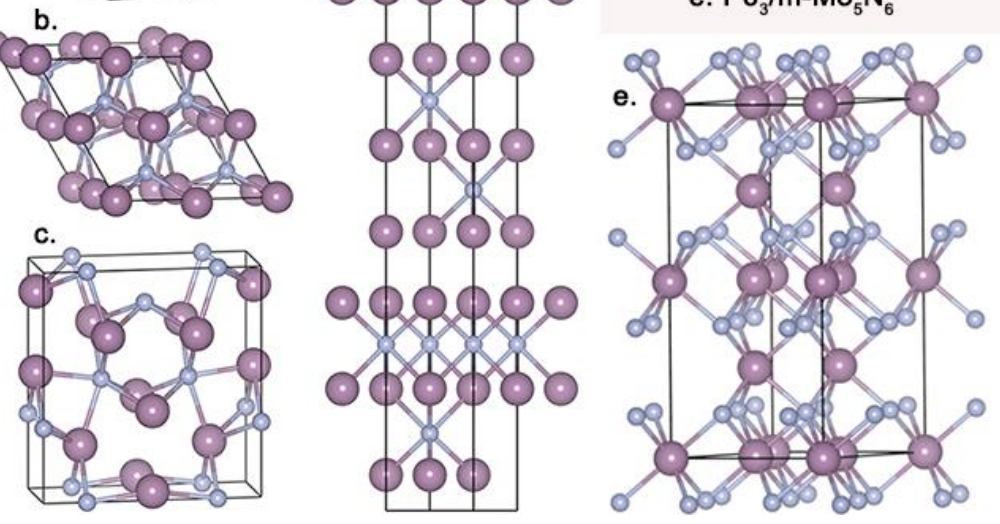
Figure $\mathbf{S 6}$ Comparison of the computed XRD patterns with experimental data.

It should be pointed out that though $\mathrm{P}_{3} / \mathrm{mmc}$ and $\mathrm{P}_{4} / \mathrm{mbm}$ structures are predicted to be much more stable than $\mathrm{R}_{3} \mathrm{~m} \mathrm{MoN}_{2}$, their lattice constants are very different from the experimental results. Even if R3m phase is dynamically, mechanically and thermodynamically unstable, we have generated the XRD pattern of a fully optimized R3m phase with considering van der Waals correction (X-ray wavelength $\lambda=1.54 \AA$ ), shown in Fig. S6. We notice that there are still some mismatches to the experimental data. Hence, based on our structural and thermodynamic analysis, we cannot get the conclusion that the synthesized compound by Wang et al. has the $\mathrm{MoS}_{2}$-type R3m structure, neither the ground-state $\mathrm{P}_{3} / \mathrm{mmc}$ pernitride one. Therefore, we hypothesize that the synthesized compound does not have the 1:2 composition, and requires further exploration.

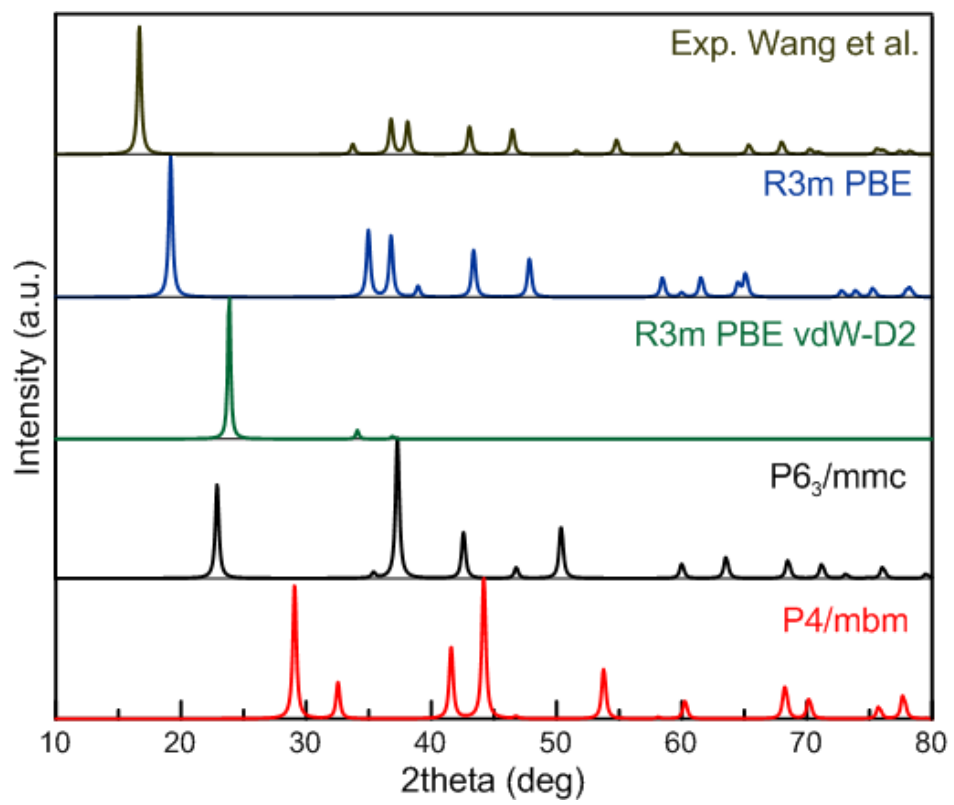

A similar discrepancy of structure and/or stoichiometry between experiments and theoretical calculations can be also found in numerous cases as in Pt-N, W-B, and Ru-C binary phases. From experiments, the Pt-N phase was initially proposed to have the 1:1 stoichiometry. ${ }^{1}$ However, first-principles calculations found that the optimized lattice parameters of the dynamically stable $\mathrm{NaCl}$-type structure of $\mathrm{PtN}$ disagree with the experimental results. ${ }^{2}$ Subsequently, both experimental and theoretical studies agreed that the actual stoichiometry is $\mathrm{PtN}_{2}{ }^{3-4}$ For $\mathrm{W}-\mathrm{B}$ compound, $\mathrm{WB}_{3}{ }^{5}$ was finally determined instead of initially experimentally proposed $\mathrm{WB}_{4}{ }^{6}{ }^{\text {For }} \mathrm{Ru}-\mathrm{C}$ compound, theoretical calculations proposed the experimental synthesized $\mathrm{Ru}-\mathrm{C}$ compound has a stoichiometry other than $\mathrm{Ru}_{2} \mathrm{C}$ or is even a mixed phase. ${ }^{7}$ Further investigations are demanded to validate our proposal that the experimentally synthesized Mo-N compound has a stoichiometry other than 1:2. Nitrogen vacancies may play an important effect on the structural stability of this compound, just as in $\operatorname{ReN}_{2}{ }^{8}$ 
Table S1 Calculated Structural Parameters, Enthalpies and Magnetic Moments of MoS $_{2}$-Like R3m $\mathrm{MoN}_{2}$ Structure. Distances Are Given in $\AA$, Energies in eV/atom, Magnetic Moments in $\mu_{B}$.

\begin{tabular}{|c|c|c|c|c|c|c|c|c|c|}
\hline Model & $\begin{array}{l}\text { Spin } \\
\text { state }\end{array}$ & $\mathrm{a}$ & C & $\begin{array}{c}\text { Interlayer } \\
\text { Mo-N } \\
\text { length }\end{array}$ & $\begin{array}{c}\text { Interlayer } \\
\text { N-N } \\
\text { length }\end{array}$ & $\begin{array}{l}\text { Mo-Mo } \\
\text { in layer }\end{array}$ & $\begin{array}{c}\text { Mo-N } \\
\text { in layer }\end{array}$ & E & $\mu_{\mathrm{B}}$ \\
\hline \multirow{3}{*}{$\begin{array}{c}\text { unrelaxed } \\
\text { PBE }\end{array}$} & NSP & 2.854 & 15.938 & 4.112 & 3.438 & 2.854 & $1.978 / 2.039$ & -8.569 & \\
\hline & FM & 2.854 & 15.938 & 4.112 & 3.438 & 2.854 & $1.978 / 2.039$ & -8.606 & 3.982 \\
\hline & AFM & 2.854 & 15.938 & 4.112 & 3.438 & 2.854 & $1.978 / 2.039$ & -8.606 & 3.982 \\
\hline \multirow{3}{*}{$\begin{array}{c}\text { only relax } \\
\text { atom } \\
\text { position } \\
\text { PBE }\end{array}$} & NSP & 2.854 & 15.938 & 4.145 & 3.385 & 2.854 & $2.020 / 2.031$ & -8.793 & \\
\hline & FM & 2.854 & 15.938 & 4.151 & 3.413 & 2.854 & $2.016 / 2.017$ & -8.830 & 4.215 \\
\hline & AFM & 2.854 & 15.938 & 4.151 & 3.413 & 2.854 & 2.016/2.017 & -8.830 & 4.215 \\
\hline \multirow{3}{*}{$\begin{array}{l}\text { Full relaxed } \\
\text { PBE }\end{array}$} & NSP & 3.010 & 13.047 & 3.256 & 2.781 & 3.010 & $2.048 / 2.052$ & -8.698 & \\
\hline & FM & 2.997 & 15.683 & 4.119 & 3.473 & 2.997 & $2.055 / 2.055$ & -8.722 & 3.842 \\
\hline & AFM & 2.997 & 15.683 & 4.119 & 3.473 & 2.997 & $2.055 / 2.055$ & -8.722 & 3.842 \\
\hline \multirow{3}{*}{$\begin{array}{l}\text { Full relaxed } \\
\text { PBE+vdW }\end{array}$} & NSP & 3.123 & 11.187 & 2.541 & 2.487 & 3.123 & $1.984 / 2.159$ & -8.993 & \\
\hline & FM & 3.122 & 11.197 & 2.545 & 2.487 & 3.122 & $1.985 / 2.159$ & -8.991 & \\
\hline & AFM & 3.122 & 11.197 & 2.545 & 2.487 & 3.122 & $1.985 / 2.159$ & -8.991 & \\
\hline \multirow{3}{*}{$\begin{array}{l}\text { Full relaxed } \\
\text { PW91 }\end{array}$} & NSP & 3.105 & 11.909 & 2.781 & 2.622 & 3.105 & $1.991 / 2.151$ & -8.725 & \\
\hline & FM & 3.004 & 15.651 & 4.113 & 3.474 & 3.004 & $2.055 / 2.056$ & -8.727 & 3.598 \\
\hline & AFM & 3.004 & 15.651 & 4.113 & 3.474 & 3.004 & $2.055 / 2.056$ & -8.727 & 3.598 \\
\hline \multirow{3}{*}{$\begin{array}{l}\text { Full relaxed } \\
\text { PW91+vdW }\end{array}$} & NSP & 3.123 & 11.047 & 2.493 & 2.453 & 3.123 & $1.985 / 2.160$ & -9.122 & \\
\hline & FM & 3.123 & 11.047 & 2.493 & 2.453 & 3.123 & $1.985 / 2.160$ & -9.122 & \\
\hline & AFM & 3.123 & 11.047 & 2.493 & 2.453 & 3.123 & $1.985 / 2.160$ & -9.122 & \\
\hline \multirow{2}{*}{$\begin{array}{l}\text { 2D slab full } \\
\text { relaxed } \\
\text { PBE+vdW }\end{array}$} & NSP & & & & & & & -8.656 & \\
\hline & FM & & & & & & & -8.720 & 1.345 \\
\hline
\end{tabular}

Theory: PAW PBE-GGA without van der Waals correction. In parentheses are given the PAW PBE-GGA with van der Waals correction (D2 Grimme method) values. See Methodology section. NSP: non spin-polarized calculation (non-magnetic phase)

FM: spin-polarized calculation (ferromagnetic phase).

AFM: spin-polarized calculation (antiferromagnetic phase). Antiferromagnetic phases have been computed using a double unit cell $\left(\mathrm{Z}=6\right.$, six $\mathrm{MoN}_{2}$ slabs/unit cell) and a tiny energy difference is found between antiferromagnetic and ferromagnetic phases at the DFT-PBE level of theory, less than $2 \mathrm{meV}$ /atom, in favour of ferromagnetic phase.

Full relaxation: both unit cells and atomic positions were relaxed.

PW91 DFT level: we used here the DFT functional chosen by Wu F. et al, Nano Lett. 15, 8277 (2015). Note that in their study, they did not optimized the a, b and c lattices (partial optimization: atomic position relaxation and fixed experimental lattices).

2D slab: we optimized the ${ }_{2} \mathrm{D}{ }_{3} \mathrm{R}-\mathrm{MoN}_{2}$ slab, both a, b lattices and atomic positions relaxation. Two slabs are separated by a vacuum of $10 \AA$. This phase is ferromagnetic but dynamically unstable. Moreover, a more stable $2 \mathrm{D} 1 \mathrm{~T} \mathrm{MoN}_{2}$ phase has been proposed recently (Mo in octahedral site), see Physics Letters A 380, 768-772. 
Table S2 Computed Enthalpies of Formation of Representative Metal Nitrides $\mathrm{M}_{\mathrm{x}} \mathrm{N}_{\mathrm{y}}$ at o $\mathrm{K}$ and o $\mathrm{GPa}(\mathrm{eV} / \mathrm{atom}, \mathrm{GPa})$.

\begin{tabular}{|c|c|c|c|c|c|}
\hline Phase & SG & $\Delta \mathrm{H}_{\mathrm{f}}$ & $\mathrm{P}$ for negative $\Delta \mathrm{H}_{\mathrm{f}}$ & B & Reference \\
\hline \multicolumn{6}{|c|}{ Nitrides } \\
\hline $\mathrm{Mo}_{2} \mathrm{~N}$ & I41/amd & -0.357 & & & Ettmayer $^{9}$ \\
\hline $\mathrm{MoN}$ & $\mathrm{P}_{3} \mathrm{mc}$ FeS $(2 \mathrm{H})$ & -0.548 & & 345 & Bull $^{10}$ \\
\hline $\mathrm{Hf}_{3} \mathrm{~N}_{4}$ & $\mathrm{I}-43 \mathrm{~d} \mathrm{Th}_{3} \mathrm{P}_{4}$ & -1.815 & & 260 & Zerr $^{11}$ \\
\hline $\mathrm{Ti}_{2} \mathrm{~N}$ & $\mathrm{P}_{42} / \mathrm{mnm}$ Rutile & -1.444 & & & Holmberg $^{12}$ \\
\hline \multicolumn{6}{|c|}{ Pernitrides } \\
\hline $\mathrm{OsN}_{2}$ & Pnnm marcasite & 0.588 & 8.5 & 387 & Wang $^{13}$ \\
\hline $\mathrm{IrN}_{2}$ & $\mathrm{P}_{2} / \mathrm{c} \mathrm{CoSb} \mathrm{C}_{2}$ & 0.451 & 3 & 373 & Chen $^{14}$ \\
\hline $\mathrm{PtN}_{2}$ & Pa-3 pyrite & 0.637 & 15 & 354 & Åberg $^{15}$ \\
\hline \multicolumn{6}{|c|}{ Nitrogen-rich compounds } \\
\hline $\mathrm{RhN}_{3}$ & Im-3 Skutterudite & 0.304 & 2 & 177 & $\mathrm{Wu}^{16}$ \\
\hline $\mathrm{CoN}_{3}$ & Im-3 Skutterudite & 0.034 & 0.1 & 153 & $\mathrm{Wu}^{16}$ \\
\hline
\end{tabular}

Table S3 The Calculated Lattice Parameters ( $\AA$, ${ }^{\circ}$ ), Wyckoff Positions, Atomic Distances (Mo-Mo, Mo-N and N-N), Enthalpies and Zero-Point Energies (ZPE, eV/atom) of the Stable and Metastable Mo-N Structures at o GPa.

\begin{tabular}{|c|c|c|c|c|}
\hline Phase & Lattice parameters & Atom position & $\begin{array}{l}\text { Atomic } \\
\text { distance }\end{array}$ & $\begin{array}{c}\mathrm{H} \\
\mathrm{ZPE}\end{array}$ \\
\hline \multicolumn{5}{|c|}{ The stable ground-state Mo-N structures } \\
\hline $\begin{array}{l}\mathrm{MoN} \\
\mathrm{P}_{3} \mathrm{mc}\end{array}$ & $\begin{array}{l}a=5.778 \\
c=5.678\end{array}$ & $\begin{array}{c}\text { Mo (o, o, o.483) Mo }(0.512,0.488,0.493) \mathrm{N} \\
(0.833,0.167,0.748) \\
\mathrm{N}(0.667,0.333,0.22)\end{array}$ & $\begin{array}{c}2.884 \\
2.157 \\
2.885\end{array}$ & $\begin{array}{c}-10.016 \\
0.032\end{array}$ \\
\hline $\begin{array}{l}\mathrm{Mo}_{4} \mathrm{~N}_{3} \\
\operatorname{Imm} 2\end{array}$ & $\begin{array}{c}\mathrm{a}=2.870 \\
\mathrm{~b}=7.121 \\
\mathrm{c}=7.505\end{array}$ & $\begin{array}{c}\text { Mo (o.5, o, o.462) Mo (o.5, o, o.113) } \\
\text { Mo (o.5, o.796, o.785) } \\
\text { N (o, o.8, o) N (o, o, o.675) }\end{array}$ & $\begin{array}{l}2.619 \\
2.162 \\
2.889\end{array}$ & $\begin{array}{l}-10.203 \\
0.063\end{array}$ \\
\hline \multicolumn{5}{|c|}{ The metastable Mo-N structures } \\
\hline $\begin{array}{l}\mathrm{MoN}_{2} \\
\mathrm{P}-6 \mathrm{~m} 2\end{array}$ & $\begin{array}{l}a=2.920 \\
c=3.901\end{array}$ & $\begin{array}{c}\text { Mo }(\mathrm{o}, \mathrm{o}, \mathrm{o}) \\
\mathrm{N}(\mathrm{o.333}, 0.667,0.678)\end{array}$ & $\begin{array}{l}2.920 \\
2.102 \\
1.392\end{array}$ & $\begin{array}{l}-9.496 \\
0.099\end{array}$ \\
\hline $\begin{array}{l}\mathrm{MoN}_{2} \\
\mathrm{P}_{2} / \mathrm{m}\end{array}$ & $\begin{array}{c}a=4.252, b=2.890 \\
c=5.212, \beta=113.9\end{array}$ & $\begin{array}{l}\text { Mo (o.011, 0.25, 0.717) } \\
\text { N (o.363, 0.75, 0.705) } \\
\text { N (0.706, 0.75, o.827) }\end{array}$ & $\begin{array}{l}2.657 \\
2.100 \\
1.332 \\
\end{array}$ & $\begin{array}{l}-9.316 \\
0.089\end{array}$ \\
\hline $\begin{array}{l}\mathrm{MoN}_{2} \\
\mathrm{R}-3 \mathrm{~m}\end{array}$ & $\begin{array}{l}a=2.904 \\
c=11.892\end{array}$ & $\begin{array}{c}\text { Mo }(\mathrm{o}, \mathrm{o}, \mathrm{o}) \\
\mathrm{N}(\mathrm{o}, \mathrm{o}, 0.556)\end{array}$ & $\begin{array}{r}2.904 \\
2.134 \\
1.323\end{array}$ & $\begin{array}{l}-9.275 \\
0.081\end{array}$ \\
\hline $\begin{array}{l}\mathrm{MoN}_{2} \\
\mathrm{C}_{2} / \mathrm{m}\end{array}$ & $\begin{array}{c}a=6.739, b=3.083 \\
c=8.110, \beta=137.0\end{array}$ & $\begin{array}{c}\text { Mo (o.941, o.5, 0.776) } \\
\mathrm{N}(0.211,0,0.85) \\
\mathrm{N}(0.129,0.5,0.536)\end{array}$ & $\begin{array}{l}3.083 \\
2.029 \\
1.377\end{array}$ & $\begin{array}{l}-9.258 \\
0.087\end{array}$ \\
\hline $\begin{array}{c}\mathrm{MoN}_{2} \\
\mathrm{P}_{3} / \mathrm{mmc}\end{array}$ & $\begin{array}{l}a=2.887 \\
c=8.004\end{array}$ & $\begin{array}{c}\text { Mo }(\mathrm{o}, \mathrm{o}, \mathrm{o}) \\
\mathrm{N}(\mathrm{o} .333,0.667,0.833)\end{array}$ & $\begin{array}{l}2.887 \\
2.135 \\
1.334\end{array}$ & $\begin{array}{l}-9.250 \\
0.081\end{array}$ \\
\hline $\begin{array}{l}\mathrm{MoN}_{2} \\
\mathrm{P}_{42} \mathrm{~nm}\end{array}$ & $\begin{array}{l}a=4.882 \\
c=3.044\end{array}$ & $\begin{array}{c}\text { Mo }(0,0,0) \\
\mathrm{N}(0.296,0.704,0.881)\end{array}$ & $\begin{array}{l}3.044 \\
1.821\end{array}$ & $\begin{array}{l}-9.165 \\
0.078\end{array}$ \\
\hline
\end{tabular}


Supporting Information

\begin{tabular}{|c|c|c|c|c|}
\hline & & & 2.810 & \\
\hline $\begin{array}{l}\mathrm{MoN}_{2} \\
\text { Pnma }\end{array}$ & $\begin{array}{l}a=5.797 \\
b=3.628 \\
c=9.731\end{array}$ & $\begin{array}{l}\text { Mo }(0.542,0.75,0.373) \\
N(0.625,0.25,0.437) \\
N(0.68,0.25,0.75)\end{array}$ & $\begin{array}{l}3.099 \\
1.976 \\
2.631\end{array}$ & $\begin{array}{l}-9.140 \\
0.074\end{array}$ \\
\hline $\begin{array}{c}\mathrm{MoN}_{2} \\
\mathrm{P}_{4} / \mathrm{mbm}\end{array}$ & $\begin{array}{l}a=4.343 \\
c=2.752\end{array}$ & $\begin{array}{c}\text { Mo }(0,0,0) \\
N(0.111,0.611,0.5)\end{array}$ & $\begin{array}{r}2.752 \\
2.231 \\
1.369\end{array}$ & $\begin{array}{l}-9.089 \\
0.089\end{array}$ \\
\hline $\begin{array}{c}\mathrm{MoN}_{2} \\
\mathrm{Cm}\end{array}$ & $\begin{array}{l}a=5.521, b=3.014 \\
c=5.822, \beta=111.5\end{array}$ & $\begin{array}{c}\text { Mo }(0,0,0) \\
N(0.154,0.5,0.202) \\
N(0.709,0.5,0.812)\end{array}$ & $\begin{array}{l}3.014 \\
2.182 \\
2.667\end{array}$ & $\begin{array}{l}-8.885 \\
0.070\end{array}$ \\
\hline $\begin{array}{c}\mathrm{MoN}_{2} \\
\mathrm{P}_{3} / \mathrm{mmc}\end{array}$ & $\begin{array}{l}a=3.052 \\
c=7.806\end{array}$ & $\begin{array}{l}\text { Mo }(0.667,0.333,0.75) \\
N(0.333,0.667,0.889)\end{array}$ & $\begin{array}{l}3.052 \\
2.067 \\
2.163\end{array}$ & $\begin{array}{l}-8.776 \\
0.070\end{array}$ \\
\hline $\begin{array}{l}\mathrm{Mo}_{2} \mathrm{~N} \\
\mathrm{Pmmn}\end{array}$ & $\begin{array}{l}a=4.203 \\
b=2.904 \\
c=5.935\end{array}$ & $\begin{array}{l}\text { Mo }(o, 1,0.116) \\
\text { Mo }(0,1,0.663) \\
N(0.5,1,0.623)\end{array}$ & $\begin{array}{l}2.871 \\
2.126 \\
2.904\end{array}$ & $\begin{array}{c}-10.355 \\
0.057\end{array}$ \\
\hline $\begin{array}{l}\mathrm{Mo}_{3} \mathrm{~N}_{2} \\
\mathrm{R}-3 \mathrm{~m}\end{array}$ & $\begin{array}{l}a=2.864 \\
c=23.901\end{array}$ & $\begin{array}{r}\text { Mo }(0,0,0.5) \\
\text { Mo }(0,0,0.618) \\
\text { N (o, o, o.772) }\end{array}$ & $\begin{array}{c}2.864 \\
2.141 \\
2.864\end{array}$ & $\begin{array}{l}-10.242 \\
0.056\end{array}$ \\
\hline $\begin{array}{l}\mathrm{Mo}_{5} \mathrm{~N}_{6} \\
\mathrm{P}_{3} / \mathrm{m}\end{array}$ & $\begin{array}{l}a=4.898 \\
c=11.199\end{array}$ & $\begin{array}{c}\text { Mo (o, o, o) Mo }(0.333,0.667,0.25) \\
\text { Mo }(0.667,0.333,0.25) \operatorname{Mo}(0.333,0.667,1) \mathrm{N} \\
(\mathrm{o}, 0.339,0.125)\end{array}$ & $\begin{array}{l}2.828 \\
2.172 \\
2.800\end{array}$ & $-9 \cdot 774$ \\
\hline
\end{tabular}

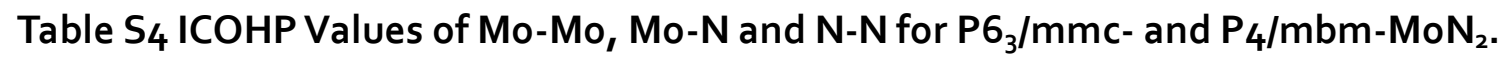

\begin{tabular}{|c|c|c|c|c|}
\hline Phase & $\mathrm{P}(\mathrm{GPa})$ & Mo-Mo & Mo-N & N-N \\
\hline $\mathrm{P}_{3} / \mathrm{mmc}$ & $\mathrm{o}$ & 0.576 & 6.890 & 6.689 \\
\hline $\mathrm{P} 4 / \mathrm{mbm}$ & 100 & 2.316 & 4.421 & 8.157 \\
\hline
\end{tabular}

\section{References}

(1) Gregoryanz, E.; Sanloup, C.; Somayazulu, M.; Badro, J.; Fiquet, G.; Mao, H. K.; Hemley, R. J., Synthesis and Characterization of a Binary Noble Metal Nitride. Nat. Mater. 2004, 3, 294-297.

(2) Patil, S. K. R.; Khare, S. V.; Tuttle, B. R.; Bording, J. K.; Kodambaka, S., Mechanical Stability of Possible Structures of Ptn Investigated Using First-Principles Calculations. Phys. Rev. B 2006, 73, 104118.

(3) Crowhurst, J. C.; Goncharov, A. F.; Sadigh, B.; Evans, C. L.; Morrall, P. G.; Ferreira, J. L.; Nelson, A. J., Synthesis and Characterization of the Nitrides of Platinum and Iridium. Science 2006, 311, 1275-1278.

(4) Yu, R.; Zhang, X. F., Platinum Nitride with Fluorite Structure. Appl. Phys. Lett. 2005, 86, 121913.

(5) Zhang, R. F.; Legut, D.; Lin, Z. J.; Zhao, Y. S.; Mao, H. K.; Veprek, S., Stability and Strength of Transition-Metal Tetraborides and Triborides. Phys. Rev. Lett. 2012, 108, 255502.

(6) Gu, Q. F.; Krauss, G.; Steurer, W., Transition Metal Borides: Superhard Versus Ultra-Incompressible. Adv. Mater. 2008, 20, 3620-3626.

(7) Lu, J.; Hong, F.; Lin, W. J.; Ren, W.; Li, Y. W.; Yan, Y. F., Novel Ultra-Incompressible Phases of Ru ${ }_{2}$ C. J. Phys.: Condens. Matter 2015, 27, 175505.

(8) Wang, Y. C.; Yao, T. K.; Yao, J. L.; Zhang, J. W.; Gou, H. Y., Does the Real $\mathrm{ReN}_{2}$ Have the $\mathrm{MoS}_{2}$ Structure? PCCP 2013, 15, 183-187.

(9) Ettmayer, P., Das System Molybdän-Stickstoff. Monatsh. Chem. 1970, 101, 127-140.

(10) Bull, C. L.; McMillan, P. F.; Soignard, E.; Leinenweber, K., Determination of the Crystal Structure of $\delta$-MoN by Neutron Diffraction. J. Solid State Chem. 2004, 177, 1488-1492.

(11) Zerr, A.; Miehe, G.; Riedel, R., Synthesis of Cubic Zirconium and Hafnium Nitride Having $\mathrm{Th}_{3} \mathrm{P}_{4}$ Structure. Nat. Mater. 2003, 2, 185-189. 


\section{Supporting Information}

(12) Holmberg, B., Structural Studies on the Titanium-Nitrogen System. Acta Chem. Scand. 1962, 16, 1255-1261. (13) Wang, Z. H.; Kuang, X. Y.; Zhong, M. M.; Lu, P.; Mao, A. J.; Huang, X. F., Pressure-Induced Structural Transition of $\mathrm{OsN}_{2}$ and Effect of Metallic Bonding on Its Hardness. EPL 2011, 95, 66005.

(14) Chen, W.; Tse, J. S.; Jiang, J. Z., An Ab Initio Study of 5 d Noble Metal Nitrides: $\mathrm{OsN}_{2}, \operatorname{IrN}_{2}, \mathrm{PtN}_{2}$ and $\mathrm{AuN}_{2}$. Solid State Commun. 2010, 150, 181-186.

(15) Åberg, D.; Sadigh, B.; Crowhurst, J.; Goncharov, A. F., Thermodynamic Ground States of Platinum Metal Nitrides. Phys. Rev. Lett. 2008, 100, 095501.

(16) $\mathrm{Wu}, \mathrm{Z}$. J.; Meng, J., Ab Initio Study on the Physical Properties of $\mathrm{CoN}_{3}$ and $\mathrm{RhN}_{3}$ with Skutterudite Structure. Comput. Mater. Sci. 2oo8, 43, 495-500. 